\title{
On a Transcendental Equation in the Stability Analysis of a Population Growth Model
}

\author{
H.-O. Walther, München
}

\begin{abstract}
Summary
We consider the rate equation $\dot{n}=r n$ for the density $n$ of a single species population in a constant environment. We assume only that there is a positive constant solution $n^{*}$, that the rate of increase $r$ depends on the history of $n$ and that $r$ decreases for great $n$. The stability properties of the solution $n^{*}$ depend on the location of the eigenvalues of the linearized functional differential equation. These eigenvalues are the complex solutions $\lambda$ of the equation $\lambda+\alpha \int_{-1}^{0} \exp [\lambda a] d s(a)=0$ with $\alpha>0$ and $s$ increasing, $s(-1)=0, s(0)=1$. We give conditions on $\alpha$ and $s$ which ensure that all eigenvalues have negative real part, or that there are eigenvalues with positive real part. In the case of the simplest smooth function $s(s=i d+1)$, we obtain a theorem which describes the distribution of all eigenvalues in the complex plane for every $\alpha>0$.
\end{abstract}

1. A population living in a constant environment cannot increase at constant rate. In laboratory experiments, the population density $n$ tends to a limit $n^{*}$, or it shows undamped oscillations (see e.g. Halbach et al., 1972). A simple and general model in accordance with these observations is the functional differential equation

$$
\dot{n}(t)=r\left(n_{t}\right) n(t), t>0
$$

for the population density $n:[-1, \infty) \rightarrow R_{0}^{+}$. Here the rate of increase is given by a real-valued mapping $r$ defined on the set of non-negative functions on the interval $[-1,0]$, with the only properties

and

$$
r\left(n^{*}\right)=0 \text { for a positive constant function } n^{*}
$$

$$
\exists \varepsilon>0: n^{*}-\varepsilon \leq \varphi \leq \psi \Rightarrow r(\psi) \leq r(\varphi) .
$$

The function $n_{t}$ is defined by $n_{t}(a):=n(t+a)$ for $-1 \leq a \leq 0$ and $t \geq 0$. - The dependence of $r$ on the density in the past allows oscillations (see e.g. Wright $1955)$, and $(R 1)$ and $(R 2)$ ensure that high densities result in a decay of the population size - which is a natural assumption. - The first model of this type (with $r(\varphi)=b(K-\varphi(-1)) / K, b$ and $K$ positive) was proposed by G. E. Hutchinson in 1948. 
We are interested in the stability of the constant solution defined by $n^{*}$. (This solution and its value are called $n^{*}$, too.) In this paper, we investigate the eigenvalues of the corresponding linearization of equation (1). Suppose in addition that

the Fréchet-derivative $\operatorname{Dr}\left(n^{*}\right)$ of $r$ in $n^{*}$ exists

(with respect to the supremum-norm on the continuous functions on $[-1,0]$ ). With $z:=n-n^{*}$ and $H(\varphi):=n^{*} r\left(\varphi+n^{*}\right)+\varphi(0) r\left(\varphi+n^{*}\right)$, equation (1) implies $\dot{z}(t)=H\left(z_{t}\right)$, and the linearization near $n^{*}$ is $\dot{y}(t)=D H(0)\left(y_{t}\right)=n^{*} \operatorname{Dr}\left(n^{*}\right)\left(y_{t}\right)$ since the derivative of the second term of $H$ vanishes. By $(R 1)$ and $(R 2)$, $\operatorname{Dr}\left(n^{*}\right)(\varphi) \leq 0$ for $\varphi \geq 0$ and continuous. Hence $n^{*} \operatorname{Dr}\left(n^{*}\right)(\varphi)=-\alpha \int_{-1}^{0} \varphi(a) d s(a)$ for all continuous functions $\varphi:[-1,0] \rightarrow R$, with $\alpha=n^{*}\left\|\operatorname{Dr}\left(n^{*}\right)\right\|$ and $s \in S:=$ $=\{\sigma:[-1,0] \rightarrow R \mid \sigma$ increasing. $\sigma(-1)=0, \sigma(0)=1\}$.

The parameter $\alpha$ may serve as a measure of the power of the negative feedback in our system. The function $s$ indicates how $\operatorname{Dr}\left(n_{*}^{*}\right)(\varphi)$ - or $r(\varphi)$ for $\varphi$ near $n^{*}$ depends on the values of $\varphi$ at the different times in the past. For example, let $r(\varphi)=b-d(\varphi)=b-\alpha \cdot \int_{-1}^{0} \varphi(a) d s(a)$, where $b \in R^{+}$stands for the birth rate and $d$ for the death rate. Then $s$ concave means that $r\left(n_{t}\right)$ is influenced more by $n \mid[-1,-1 / 2]$ than by $n \mid[-1 / 2,0]$. - One might expect that for $s$ concave the stability of $n^{*}$ is in some way less than for $s$ convex because in the first case the system takes longer to produce a sufficient reaction to a perturbation of the equilibrium $n^{*}$. We shall see below in which way this conjecture turns out to be right.

The linearized equation becomes

$$
\dot{y}(t)=-\alpha \int_{-1}^{0} y \cdot(t+a) d s(a) .
$$

The eigenvalues of equation (2) are the complex solutions of the transcendental equation

$$
\lambda+\alpha \int_{-1}^{0} \exp [\lambda a] d s(a)=0
$$

or, in other words, the zeros of the entire function $f(\cdot, \alpha, s): \lambda \mapsto \lambda+\alpha \int_{-1}^{0} \exp [\lambda a] d s(a)$. The zero solution of equation (2) and $n^{*}$ are asymptotically stable if all eigenvalues lie in the left half-plane $C^{-}:=R^{-}+i R$ (see [3], chapter 22, and [5]). If one eigenvalue is in $C^{+}:=R^{+}+i R$ then the zero solution of equation (2) is unstable, and for $r(\varphi)=\alpha-\varphi(-1), n^{*}(=\alpha)$ is unstable too (Wright 1955).

Due to [5], we have

$$
0<\alpha<\pi / 2 \wedge s \in S \wedge f(\lambda, \alpha, s)=0 \Rightarrow \lambda \in C^{-} .
$$

For the minimal (convex) function $s_{0}$ in $S$ (i.e. $s_{0}(a)=0$ for $a<0$ ), the equation for the eigenvalues reduces to $\lambda+\alpha=0$, hence $\lambda \in C^{-}$for all $\alpha>0$. In the case of the maximal (concave) functions $s_{1}$ in $S$ (i.e. $s_{1}(a)=1$ for $-1<a$ ) there exist $\alpha>0$ such that at least one eigenvalue is in $C^{+}$(in fact, $\alpha>\pi / 2$ is sufficient), see (Wright 1955). 
Theorem 2 in section 2 shows that this property of $s_{0}$ carries over to a class of smooth convex functions in $S$. In Theorem 3 and Theorem 4 we present a class $A$ of functions $s$ in $S$ with eigenvalues in $C^{+}$for certain $\alpha$, like $s_{1}$. The class $A$ contains every concave function and all $s \geq i d+1$ which are continuously differentiable.

The proof that $n^{*}$ is unstable for $r(\varphi)=\alpha-\varphi(-1)$ and $\alpha>\pi / 2$ requires - apart from the existence of an eigenvalue $\lambda=u+i v$ of the linearized equation in $C^{+}-$ the estimate $|v|<\pi$. Therefore we examine the boundedness of the branches of the eigenvalues in $C^{+}$for given functions $s$ (Theorem 5).

Section 3 deals with the eigenvalues for the simplest smooth function in $S$, that is $s=i d+1$. Theorem 6 describes the location of the eigenvalues in $C$ for all $\alpha>0$. In particular we see that $|v|>\pi$ for every eigenvalue in $C^{+}$.

2. In the following, we always assume $\alpha>0$ and $s \in S \mid\left\{s_{0}\right\}$. For a subset $M \subset C$, $Z(\alpha, s, M)$ denotes the number of zeros of $f(\cdot, \alpha, s)$ in $M$. For $\lambda \in C$, we write $\lambda=u+i v$ with $u$ and $v$ real. We have

$$
\begin{gathered}
f(\lambda, \alpha, s)=0 \Leftrightarrow u+\alpha \int_{-1}^{0} \exp [u a] \cos v a d s(a)=0 \wedge \\
v+\alpha \int_{-1}^{0} \exp [u a] \sin v a d s(a)=0, \\
f(\lambda, \alpha, s)=0 \Leftrightarrow f(\bar{\lambda}, \alpha, s)=0, \\
f(\lambda, \alpha, s)=0 \wedge u \geq 0 \Rightarrow|\lambda| \leq \alpha \wedge v \neq 0 \text { (in particular } f(0, \alpha, s) \neq 0) .
\end{gathered}
$$

First, we consider real eigenvalues. Set $g(u, s):=-u^{\prime} \int_{-1}^{0} \exp [u a] d s(a)$ for $u \leq 0$.

Theorem 1: There are real eigenvalues if and only if $\alpha \leq \max g(\cdot, s)$. Every real eigenvalue is negative.

Proof: $s \in S, s \neq s_{0}$ and $u \leq 0$ imply $\int_{-1}^{0} \exp [u a] d s(a) \geq \int_{-1}^{-\varepsilon} \exp [u a] d s(a) \geq$ $\geq \exp [-\varepsilon u] s(-\varepsilon)>0$ for small $\varepsilon>0$, hence $g(u, s) \rightarrow 0$ for $u \rightarrow-\infty$. By $g(0, s)=0$, $\max g(\cdot, s)$ exists. By $(4), u \in R \wedge f(u, \alpha, s)=0 \Leftrightarrow \alpha=g(u, s) \wedge u<0$. This implies Theorem 1.

Theorem 2: Let $s \in C^{2}[-1,0] \cap C^{3}(-1,0]$ be given with $s^{\prime}(-1)=0, s^{\prime \prime}(-1)=0$, $s^{\prime \prime \prime} \geq 0, s^{\prime \prime \prime}\left(a^{*}\right)>0$ for a certain $a^{*} \in(-1,0)$. Then for every $\alpha>0$, every eigenvalue has negative real part.

Proof: a) No eigenvalue on $i R$ : By (4), (5) and $u=0$, we only have to show $\int_{-1}^{0} \cos v a d s(a)>0$ for all $v>0$. Let $v>0$. With $s^{\prime}(-1)=0, \int_{-1}^{0} \cos v a d s(a)=$ $=-(1 / v) \int_{-1}^{0} s^{\prime \prime}(a) \sin v a d a .-\int_{-1}^{0} s^{\prime \prime}(a) \sin v a d a=\lim _{\varepsilon \rightarrow 0}-\int_{\varepsilon}^{0} s^{\prime \prime}(a) \sin v a d a=(1 / v)$ $\lim _{\varepsilon \rightarrow 0}\left(s^{\prime \prime}(0)-s^{\prime \prime}(\varepsilon-1) \cos (v \varepsilon-v)-\int_{\varepsilon-1}^{0} s^{\prime \prime \prime}(a) \cos v a d a\right)$. 
By $s_{0}^{\prime \prime}(-1)=0$ and $s^{\prime \prime}(0)=\int_{\varepsilon-1}^{0} s^{\prime \prime \prime}{ }_{0}(a) d a+s^{\prime \prime}(\varepsilon-1)$, the last term equals $(1 / v)$ $\lim \int_{\varepsilon}^{0} s^{\prime \prime \prime}(a)(1-\cos v a) d a \geq(1 / v) \int_{\alpha^{*}}^{0} s^{\prime \prime \prime}(a)(1-\cos v a) d a>0$.

b) No eigenvalue in $C^{+}: Z\left(\alpha, s, C^{+}\right)>0$ for $\alpha>0$ implies $\tilde{\alpha}:=\inf \{\alpha>0 \mid Z(\alpha, s$, $\left.\left.C^{+}\right)>0\right\} \in[\pi / 2, \infty)$, see (3). Then there are sequences $\alpha_{n} \rightarrow \tilde{\alpha}, \lambda_{n}$ in $C^{+}$with $f\left(\lambda_{n}, \alpha_{n} s\right)=0$. By (6), there exist subsequences $\alpha_{n}^{\prime} \rightarrow \tilde{\alpha}, \lambda_{n}^{\prime} \rightarrow \bar{\lambda} \in \bar{C}^{+}$with $f(\tilde{\lambda}, \tilde{\alpha}, s)=0$. a) gives $\lambda \in C^{+}$. Then $Z(\tilde{\alpha}, s, \partial D)=0$ for a compact disk $D$ in $C^{+}$with $\tilde{\lambda} \in \bar{D}$, and Theorem 9.17.4 of [1] guarantees the existence of $\varepsilon>0$ with $Z(\tilde{\alpha}-\varepsilon, s, D)=$ $=Z(\tilde{\alpha}, s, D)>0$, contradiction.

Examples: Theorem 2 holds for $s: a \mapsto(a+1)^{\beta}$ with $\beta>2$. The case $\beta=2$ shows that the theorem is optimal in a certain sense: The function $s: a \mapsto(a+1)^{2}$ fulfills the hypotheses except of $s^{\prime \prime \prime}\left(a^{*}\right)>0$, and one verifies easily that $f\left(2 \pi k i,(2 \pi k)^{2} / 2, s\right)$ is zero for every integer $k \neq 0$.

A class of discontinuous functions $s$ with $Z\left(\alpha, s, \overline{C^{+}}\right)=0$ for all $\alpha>0$ is defined by $\lim _{a \rightarrow 0} s(a)<1 / 2$.

Proof: Set $s^{*}(0):=\lim _{\alpha \rightarrow 0} s(a)$ and $s^{*}(a):=s(a)$ for $a<0$. Assume $f(\lambda, \alpha, s)=0$ and $u \geq 0 . B y(4), u=-\alpha \int_{-1}^{0} \exp [u a] \cos v a d s(a)=-\alpha\left(1-s^{*}(0)+\int_{-1}^{0} \exp [u a] \cos v a d s^{*}\right.$ (a)). By $\left|\int_{-1}^{0} \exp [u a] \cos v a d s^{*}(a)\right| \leq s^{*}(0)$, we obtain $u \leq-\left(1-2 s^{*}(0)\right)$, contradiction to $u \geq 0$.

Theorem 3: For $s \in A:=\{\dot{\sigma} \in S \mid \exists v \in[0, \pi] \exists \alpha>0: f(i v, \alpha, \sigma)=0\}$, there exist eigenvalues with positive real part for certain $\alpha>0$.

Proof: a) For $s \in S$, define a map $F=\left(\begin{array}{c}F_{1} \\ F_{2}\end{array}\right): R^{2} \times R^{+} \rightarrow R^{2}$ by $F_{1}(u, v, \alpha):=\operatorname{Re} f$ $(u+i v, \alpha, s), F_{2}(u, v, \alpha):=\operatorname{Im} f(u+i v, \alpha, s)$. If $F(0, v, \alpha)=0$ (which is equivalent to $f(i v, \alpha, s)=0)$ and if

$0<d:=\operatorname{det}\left(\begin{array}{cc}\frac{\partial F_{1}}{\partial u} & \frac{\partial F_{1}}{\partial v} \\ \frac{\partial F_{2}}{\partial u} & \frac{\partial F_{2}}{\partial v}\end{array}\right) \quad(0, v, \alpha)=\left(1+\alpha \int_{-1}^{0} a \cos v a d s(a)\right)^{2}+\alpha^{2}\left(\int_{-1}^{0} a \sin v a d s(a)\right)^{2}$,

then there are neighborhoods $U$ of $\alpha$ and $W$ of $(0, v)$ and a differentiable map $G=\left(G_{1}, G_{2}\right): U \rightarrow W$ with $G(\alpha)=(0, v)$ and $F \circ(G, i d)=0$. With $G(\alpha)=(0, v)$, we obtain $G_{1}^{\prime}(\alpha)=-d^{-1}\left(\frac{\partial F_{1}}{\partial \alpha} \cdot \frac{\partial F_{2}}{\partial v}-\frac{\partial F_{1}}{\partial v} \cdot \frac{\partial F_{2}}{\partial \alpha}\right)(0, v, \alpha)=d^{-1} \int_{-1}^{0} v a \sin v a d s(a)$. Therefore, Theorem 3 will follow from the existence of $\alpha>0$ and $v>0$ with $f(i v, \alpha, s)=0$ and

$$
\int_{-1}^{0} a \sin v a d s(a)>0 .
$$

b) $s \in A, f(i v, \alpha, s)=0$ and $v \in[0, \pi]$ imply $v>0$, by (6). Hence $a \sin v a>0$ for $-1<a<0$. If $v<\pi$, we have in addition $-\sin (-v)>0$, and $s \neq s_{0}$ gives (7). The 
case $v=\pi$ and $\int_{-1}^{0} a \sin v a d s(a)=0$ is impossible because $\int_{-1}^{0} a \sin \pi a d s(a)=0$ implies $s \mid(-1,0)$ constant, hence by $(4)-\pi / \alpha=\int_{-1}^{0} \sin \pi a d s(a)=s(1 / 2) \sin (-\pi)+$ $+(1-s(1 / 2)) \sin 0=0$, contradiction.

Theorem 4: For $s$ in $C^{1}[-1,0], s \in A$ is equivalent with $-\int_{-1}^{0} \pi s(a) \sin \pi a d a \geq 1$. In particular, every function $s$ in $C^{1}[-1,0]$ with $s \geq i d+1$ is in $A$. $A$ contains every concave function in $S$.

Proof: a) Let $s \in C^{1}[-1,0]$, set $h(t)=\int_{-1}^{0} \cos t a d s(a)$ for $t \geq 0$. We have $h^{\prime}(t) \leq 0$ for $0 \leq t \leq \pi$ and $h(0)=1$, hence $h(\pi) \leq 0 \Leftrightarrow \exists v \in(0, \pi]: h(v)=0$. For every $v \in(0, \pi]$ with $h(v)=0, \int_{-1}^{0} \sin v a d s(a)<0$ by $s \in C^{1}$. Then $f\left(i v,-v \int_{-1}^{0} \sin v a d s(a), s\right)=0$, by (4). We infer $(s \in A \Leftrightarrow h(\pi) \leq 0)$. Obviously, $0 \geq h(\pi)=\int_{-1}^{0} s^{\prime}(a) \cos \pi a d a=1+\int_{-1}^{0} \pi s(a)$ $\sin \pi a d a$.

b) $s \geq i d+1 \Rightarrow-\int_{-1}^{0} \pi s(a) \sin \pi a d a \geq-\int_{-1}^{0} \pi(a+1) \sin \pi a d a=1$.

c) Let $s$ be concave. For $n \in N$, set $a_{y}:=-1+v / 2 n$ for $v=0,1, \ldots, 2 n$. Then $\cos \pi a_{v-1}=-\cos \pi a_{2 n-(v-1)}$ and $s\left(a_{v}\right)-s\left(a_{v-1}\right) \geq s\left(a_{n+\mu}\right)-s\left(a_{n+\mu-1}\right)$ for $1 \leq v, \mu \leq n$. We infer $0 \geq \sum_{\kappa}^{2 n}\left(s\left(a_{\kappa}\right)-s\left(a_{\kappa-1}\right)\right) \cos a_{\kappa-1} \pi$, hence $h(\pi) \leq 0$ and $h(v)=0$

for a certain $v \in[0, \pi]$. Obviously, $\int_{-1}^{0} \sin v a d s(a) \leq 0$. $\int_{-1}^{0} \sin v a d s(a)=0$ implies $v=\pi$ (since $s \geq i d+1)$ and $s \mid(-1,0)$ constant, hence $s \mid(-1,0]=1$ and $\int_{-1}^{0} \cos v a d s(a)=-1$, contradiction. Now $s \in A$ follows as in a).

For fixed $s$, let $P$ denote the set of eigenvalues with positive real part, that is $\left\{\lambda \in C^{+} \mid \exists \alpha>0: f(\lambda, \alpha, s)=0\right\}$. In general, $P$ is unbounded, see Theorem 6 below. But we have

Theorem 5: For $s \in C^{3}[-1,0]$ with $s^{\prime}(-1)>0$ and $s^{\prime}(0)>0$, every connected subset of $P$ is bounded.

Proof: a) $f(\lambda, \alpha, s)=0$ implies $\lambda \neq 0$. Let $s \in C^{3}$. Integration by parts yields

and

$$
\left(\lambda^{2}\right) / \alpha+s^{\prime}(0)=s^{\prime}(-1) \exp [-\lambda]+\int_{-1}^{0} \exp [\lambda a] s^{\prime \prime}(a) d a
$$

$\left(\lambda^{3}\right) / \alpha+\lambda s^{\prime}(0)=s^{\prime}(-1) \exp [-\lambda]+s^{\prime \prime}(0)-\exp [-\lambda] s^{\prime \prime}(-1)-\int_{-1}^{0} \exp [\lambda a] s^{\prime \prime \prime}(a) d a$.

b) Assume $P \neq \emptyset, \operatorname{Re} P$ unbounded and $\operatorname{Im} P$ bounded. Then (8) holds for sequences $\lambda_{n}=u_{n}+i v_{n}$ in $P$ and $\alpha_{n}$ in $R^{+}$, with $u_{n} \rightarrow \infty$. (8) gives

$$
\left(u_{n}^{2}-v_{n}^{2}\right) / \alpha_{n}+s^{\prime}(0)=\operatorname{Re}\left(\lambda_{n}^{2} / \alpha_{n}+s^{\prime}(0)\right) \rightarrow 0 \text {. }
$$

By (6) and $u_{n} \rightarrow \infty, \alpha_{n} \rightarrow \infty$. Hence $v_{n}^{2} / \alpha_{n} \rightarrow 0,0<s^{\prime}(0) \leq s^{\prime}(0)+u_{n}^{2} / \alpha_{n} \rightarrow 0$, contradiction. 
b) Now let $Q$ be a connected subset of $P$. By (5) and (6), we may assume $\operatorname{Im} Q \subset R^{+}$. For $\operatorname{Im} Q$ unbounded, there exist sequences $\alpha_{n}, u_{n}$ in $R^{+}$and an integer $n_{0}$ with $f\left(u_{n}+i(2 n \pi+\pi / 2), \alpha_{n}, s\right)=0$ for $n \geq n_{0}$. Set $\lambda_{n}:=u_{n}+i(2 n \pi+\pi / 2)$. We have $\alpha_{n} \rightarrow \infty$ and $\int_{-1}^{0} \exp \left[\lambda_{n} a\right] s^{\prime \prime}(a) d a \rightarrow 0, \int_{-1}^{0} \exp \left[\lambda_{n} a\right] s^{\prime \prime \prime}(a) d a \rightarrow 0$. (Proof: It is sufficient to show the assertion in the cases $u_{n} \rightarrow \infty$ and $u_{n} \rightarrow u^{*} \geq 0$. The first case is trivial. In the second case, Theorem 4.6 of [7] gives, for example,

$\int_{-1}^{0} \exp \left[u^{*} a\right] s^{\prime \prime}(a) \cos v_{n} a d a \rightarrow 0$. In addition, $\mid \int_{-1}^{0} \exp \left[u^{*} a\right] s^{\prime \prime}(a) \cos v_{n} a d a-$ 0

$\left.-\int_{-1}^{0} \exp \left[u_{n} a\right] s^{\prime \prime}(a) \cos v_{n} a d a|\leq \max | s^{\prime \prime}|| \exp \left[-u_{n}\right]-\exp \left[-u^{*}\right] \mid \rightarrow 0.\right)-$ Assume $u_{n}$ bounded. By (8) and $\cos v_{n}=0,(10)$ holds for $\lambda_{n}$ and $\alpha_{n}$, if $n \geq n_{0}$. Hence $v_{n}^{2} / \alpha_{n} \rightarrow s^{\prime}(0)$. Then $v_{n} / \alpha_{n} \rightarrow 0$ and $2 u_{n} v_{n} / \alpha_{n} \rightarrow 0$. On the other hand, (8) yields $2 u_{n} v_{n} / \alpha_{n}-s^{\prime}(-1) \exp \left[-u_{n}\right]=\operatorname{Im}\left(\lambda_{n}^{2} / \alpha_{n}+s^{\prime}(0)-s^{\prime}(-1) \exp \left[-\lambda_{n}\right]\right) \rightarrow 0$. We infer $\exp \left[-u_{n}\right] \rightarrow 0$, contradiction.

Next, suppose $u_{n_{k}} \rightarrow \infty$ for a subsequence. Set $g_{k}:=u_{n_{k}}, h_{k}:=v_{n_{k^{\prime}}}, \beta_{k^{\prime}}:=\alpha_{n_{k^{*}}}$ Taking real and imaginary parts in (9), we obtain

$$
g_{k}\left(\left(g_{k}^{2}-3 h_{k}^{2}\right) / \beta_{k}+s^{\prime}(0)\right)-s^{\prime}(-1) \exp \left[-g_{k}\right] h_{k}-s^{\prime \prime}(0) \rightarrow 0
$$

and $h_{k}\left(\left(3 g_{k}^{2}-h_{k}^{2}\right) / \beta_{k}+s^{\prime}(0)\right) \rightarrow 0$, therefore

$$
\left(3 g_{k}^{2}-h_{k}^{2}\right) / \beta_{k}+s^{\prime}(0) \rightarrow 0 \text {. }
$$

We have $-s^{\prime}(-1) \exp \left[-g_{k}\right] h_{k} \rightarrow-\infty$. (Proof: For a subsequence $g_{k}^{\prime}+i h_{k}^{\prime}$ with $s^{\prime}(-1) \exp \left[-g_{k}^{\prime}\right] h_{k}^{\prime}$ bounded, (11) and $g_{k}^{\prime} \rightarrow \infty$ imply $\left(g_{k}^{\prime 2}-3 h_{k}^{\prime 2}\right) / \beta_{k}^{\prime}+s^{\prime}(0) \rightarrow 0$. By (12), we obtain $\left(g_{k}^{\prime 2}+h_{k}^{\prime 2}\right) / \beta_{k}^{\prime} \rightarrow 0$. By (12) again, $s^{\prime}(0)=0$, contradiction.)

Now (11) yields $0<\left(g_{k}^{2}-3 h_{k}^{2}\right) / \beta_{k}+s^{\prime}(0)$ (for $k$ large) $\leq\left(3 g_{k}^{2}-h_{k}^{2}\right) / \beta_{k}+s^{\prime}(0) \rightarrow 0$, therefore $s^{\prime}(0)=0$, contradiction.

3. Location of the eigenvalues for $s=i d+1$. We set $f_{\alpha}:=f(\cdot, \alpha, i d+1)$ and $Z(\alpha, M):=Z(\alpha, i d+1, M)$ for $\alpha>0, v_{k}:=2 k \pi+\pi$ and $\alpha_{k}:=v_{k}^{2} / 2$ for $k \in N_{0}$, $G_{k}:=R+i I_{k}$ with $I_{0}:=(-2 \pi, 2 \pi)$ and $I_{k}:=(2 k \pi, 2 k \pi+2 \pi)$ for $k \in N$, $g:=g(\cdot, i d+1), \alpha^{*}:=\max g \cdot u^{*}$ is defined by $g\left(u^{*}\right)=\alpha^{*}$. Because of $(5)$, we only consider eigenvalues with $v \geq 0$.

\section{Theorem 6:}

i) Every zero $\lambda=u+i v$ of $f_{a}, \alpha>0$, with $v \geq 0$ lies in the set $\bigcup_{k \in N_{0}} G_{k}$.

ii) For $k \in N$ and $\alpha>0, f_{\alpha}$ has exactly one zero $\lambda_{k}(\alpha)$ in $G_{k}$. We have $\lambda_{k}\left(\alpha_{k}\right)=i v_{k}$, $\lambda_{k}(\alpha) \in R^{-}+i(2 k \pi, 2 k \pi+\pi)$ for $\alpha<\alpha_{k}$ and $\lambda_{k}(\alpha) \in R^{+}+i(2 k \pi+\pi, 2 k \pi+2 \pi)$ for $\alpha>\alpha_{k}$.

iii) For every $\alpha>0, f_{\alpha}$ has exactly two zeros in $G_{0}$. These zeros are real and simple, if $\alpha<\alpha^{*}$. If we denote them by $u_{1}(\alpha)<u_{2}(\alpha)$, then $u_{1}(\alpha)<u^{*}<u_{2}(\alpha)$ and $u_{1}(\alpha) \rightarrow-\infty, u_{2}(\alpha) \rightarrow 0$ for $\alpha \rightarrow 0$. For $\alpha=\alpha^{*}, u^{*}$ is a double zero. For $\alpha^{*}<\alpha$, $f_{\alpha}$ has one zero $\lambda_{0}(\alpha)$ in $G_{0}$ with positive imaginary part. We have $\lambda_{0}(\alpha) \in R^{-}+i(0, \pi)$, if $\alpha^{*}<\alpha<\alpha_{0}, \lambda_{0}\left(\alpha_{0}\right)=i v_{0}$ and $\lambda_{0}(\alpha) \in R^{+}+i(\pi, 2 \pi)$, if $\alpha>\alpha_{0}$. 


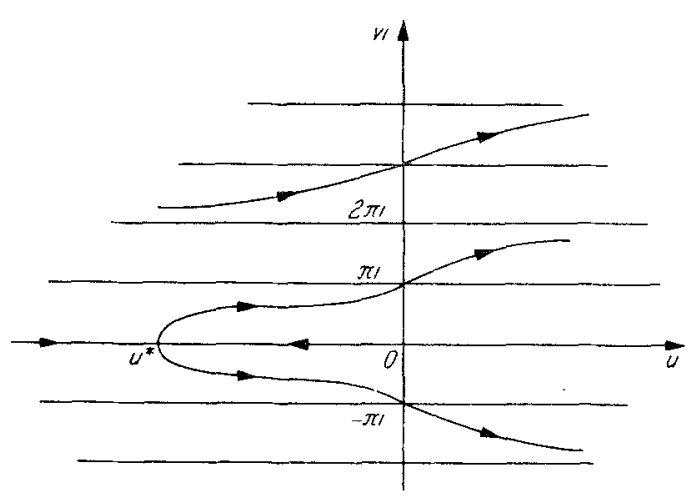

Fig. 1. The arrows indicate the direction of increasing $\alpha$

Proof of Theorem 6: We have $f_{\alpha}(\lambda)=0 \Leftrightarrow \lambda \neq 0 \wedge$

$$
\left(\lambda^{2}+\alpha\right) \exp [\lambda]=\alpha \text {. }
$$

Assertion i) is a consequence of

a) Let $f_{\alpha}(\lambda)=0, v \geq 0$. Then $v \notin 2 \pi N$, and we have

$k \in N_{0} \wedge \lambda \in G_{k} \wedge\left\{\begin{array}{l}u<0 \Rightarrow v<2 k \pi+\pi \\ u>0 \Rightarrow v>2 k \pi+\pi\end{array}\right.$

Proof: By (13), $\lambda^{2}+\alpha=\alpha \exp [-\lambda]$. Hence

$$
2 u v=\alpha \exp [-u] \sin (-v) \text {. }
$$

Therefore, $u \neq 0$ and $v \in 2 \pi N$ would imply $v=0 . u=0$ yields $v \in \pi N_{0}$. By (13), $u=0$ and $v \in 2 \pi N_{0}$ would imply $\lambda^{2}=0$, contradiction to (6). Together, we obtain $v \notin 2 \pi N$. The implication in a) is obvious from (14).

b) $f_{\alpha}(i v)=0 \wedge v \geq 0 \Leftrightarrow \exists k \in N_{0}: \alpha=\alpha_{k} \wedge v=v_{k}$.

Proof: Clearly $f_{\alpha_{k}}\left(i v_{k}\right)=0$ for $k \in N_{0}$. As in the preceding proof, we have $\left(f_{\alpha}(i v)=0 \wedge v \geq 0 \Rightarrow \exists k \in N_{0}: v=2 k \pi+\pi=v_{k}\right)$. Then (13) gives $\alpha=v^{2} / 2=v_{k}^{2} / 2=\alpha_{k}$.

c) $\alpha \leq \alpha_{k} \Rightarrow Z\left(\alpha, G_{k} \cap C^{+}\right)=0$.

Proof: Suppose $\alpha^{\prime} \leq \alpha_{k}$ and $Z\left(\alpha^{\prime}, G_{k} \cap C^{+}\right)>0$. We may assume $\alpha^{\prime}<\alpha_{k}\left(Z\left(\alpha_{k}\right.\right.$, $\left.G_{k} \cap C^{+}\right)>0$ implies $Z\left(\alpha^{\prime}, G_{k} \cap C^{+}\right)>0$ for certain $\alpha^{\prime}<\alpha_{k}$, compare part b) in the proof of Theorem 2). By (3), $\alpha^{\prime} \geq \pi / 2$. Set $B:=\left(0, \alpha^{\prime}+1\right)+i I_{k}$. We have $Z\left(\alpha^{\prime}, B\right)>0$, by (6), and $Z(\alpha, \partial B)=0$ for $1 \leq \alpha \leq \alpha^{\prime}$. This follows from a) together with (6), $\alpha<\alpha_{k}$ and with

$$
f_{\alpha}(i v)=0 \wedge i v \in G_{k} \Rightarrow \alpha=\alpha_{k} .
$$

With the aid of Theorem 9.17 .4 of $[1]$, we derive $Z(1, B)=Z\left(\alpha^{\prime}, B\right)>0$ which contradicts (3).

d) Let $k \in N_{0}$ and $\alpha>0$. There exists a negative constant $T(\alpha, k)$ with

$$
\alpha^{\prime} \geq \alpha \wedge \lambda \in G_{k} \cap C^{-} \wedge f_{\alpha^{\prime}}(\lambda)=0 \Rightarrow T(\alpha, k)<u .
$$


Proof: By (13), we have

$$
1+\left(u^{2}+v^{2}\right) / \alpha \geq\left|1+\lambda^{2} / \alpha^{\prime}\right|=|\exp [-\lambda]|=\exp [-u],
$$

therefore $u^{2} / \alpha \geq \exp [-u]-1-(2 k \pi+2 \pi)^{2} / \alpha$. This estimate and $u<0$ yield the proposition.

e) $\forall k \in N_{0} \exists \alpha_{k}^{*}>\alpha_{k}: \alpha \geq \alpha_{k}^{*} \wedge f_{\alpha}(\lambda)=0 \wedge \lambda \in G_{k} \Rightarrow u>0$.

Proof: Suppose there are sequences $\alpha(n), \lambda_{n}$ with $f_{\alpha(n)}\left(\lambda_{n}\right)=0, \alpha_{k}<\alpha(n) \rightarrow \infty$, $\lambda_{n} \in G_{k}, u_{n} \leq 0$. By (15) and $\alpha_{k}<\alpha(n), u_{n}<0$. Proposition d), (13), $u_{n}<0$ and $\alpha(n)>\alpha_{k}>1$ give

$$
\left((2 k \pi+2 \pi)^{2}+T(1, k)^{2}\right) / \alpha(n) \geq\left|\lambda_{n}\right|^{2} / \alpha(n) \geq \exp \left[-u_{n}\right]-1>0 .
$$

Therefore $\exp \left[-u_{n}\right] \rightarrow 1, u_{n} \rightarrow 0$. Together with $v_{n} \rightarrow 0$, this would imply $\int_{-1}^{0} \exp \left[\lambda_{n} a\right] d a \rightarrow 1$, hence $\left|f_{\alpha(n)}\left(\lambda_{n}\right)\right| \rightarrow \infty$, contradiction. - We obtain $\left|v_{n}\right| \geq \delta$ for a certain $\delta>0$ and for $n \in N^{*}$ with $N^{*} \subset N$ unbounded. By (13) and $u_{n}<0$, $\alpha(n)^{2} \leq\left|\lambda_{n}^{2}+\alpha(n)\right|^{2}=\alpha(n)^{2}+2 \alpha(n)\left(u_{n}^{2}-v_{n}^{2}\right)+\left|\lambda_{n}\right|^{4}$. Because of $u_{n} \rightarrow 0$, $\alpha(n) \leq\left|\lambda_{n}\right|^{4} / 2\left(v_{n}^{2}-u_{n}^{2}\right)$ for large $n \in N^{*}$, in contradiction to $\alpha(n) \rightarrow \infty$.

f) $Z\left(\alpha_{0}, G_{0}\right)=2 . \forall k \in N: Z\left(\alpha_{k}, G_{k}\right)=1$.

Proof: Let $k \in N_{0}$. The zeros $i v_{k},-i v_{k}$ of $f_{\alpha_{k}}$ are simple $\left(\lambda \neq 0 \Rightarrow f_{\alpha}(\lambda)=\right.$ $=\lambda+\alpha(1-\exp [-\lambda]) / \lambda \Rightarrow\left(d f_{\alpha} / d \lambda\right)(\lambda)=1+\alpha \exp [-\lambda] / \lambda-\alpha / \lambda^{2}+\alpha \exp [-\lambda] / \lambda^{2} \Rightarrow$ $\left.\Rightarrow\left(d f_{\alpha_{k}} / d \lambda\right)\left( \pm i v_{k}\right)=2 \pm i v_{k} / 2\right)$. Therefore, i) and b) and c) imply $Z\left(\alpha_{k}, \bar{G}_{k} \cap C^{+}\right)=1$, if $k \in N$, and $\ldots=2$ for $k=0$. Suppose $Z\left(\alpha_{k}, G_{k} \cap C^{-}\right)>0$. Then $Z\left(\alpha^{\prime}, G_{k} \cap C^{-}\right)>0$ for certain $\alpha^{\prime} \in\left(\alpha_{k}, \alpha_{k}^{*}\right)$, compare the proof of c). Set $B:=\left(T\left(\alpha_{k}, k\right), 0\right)+i I_{k}$. We have $Z\left(\alpha^{\prime}, B\right)>0$ and $Z(\alpha, \partial B)=0$ for $\alpha^{\prime} \leq \alpha \leq \alpha_{k}^{*}$, by d), a) and (15). Hence $Z\left(\alpha_{k}^{*}, B\right)=Z\left(\alpha^{\prime}, B\right)>0$, in contradiction to $\left.\mathrm{e}\right)$.

g) For every $k \in N_{0}$ and every $\alpha>0, Z\left(\alpha, G_{k}\right)=Z\left(\alpha_{k}, G_{k}\right)$.

Proof: Let $k \in N_{0}, \alpha>\alpha_{k}$ (the proof for $\alpha<\alpha_{k}$ is similar). There exists $T>0$ with

$$
\lambda \in G_{k} \wedge \alpha_{k} \leq \alpha^{\prime} \leq \alpha \wedge f_{\alpha^{\prime}}(\lambda)=0 \Rightarrow|u|<T .
$$

(Proof: The zeros with $u>0$ and $u<0$ are bounded by (6) and by d) respectively.) Set $B:=(-T, T)+i I_{k}$. For $\alpha_{k} \leq \alpha^{\prime} \leq \alpha, Z\left(\alpha^{\prime}, \partial B\right)=0$. Hence $Z(\alpha, B)=Z\left(\alpha_{k}, B\right)$, and (16) implies $\mathrm{g})$.

h) Let $k \in N_{0}$ and $\alpha<\alpha_{k}$. By c), $u \leq 0$ for the zeros of $f_{\alpha}$ in $G_{k}$. (15) yields $u<0$. By a), $2 k \pi<v<2 k \pi+\pi$ for $k \in N$ and $|v|<\pi$ for $k=0$.

j) For $k \in N_{0}$ and $\alpha>\alpha_{k}$, set $B:=(T(\alpha, k), 0)+i I_{k}$. e) implies the existence of $\alpha_{k}^{* *} \geq \alpha$ with $Z\left(\alpha_{k}^{* *}, B\right)=0$. By d) and (15), $Z\left(\alpha^{\prime}, \partial B\right)=0$ for $\alpha \leq \alpha^{\prime} \leq \alpha_{k}^{* *}$. Hence $Z(\alpha, B)=Z\left(\alpha_{k}^{* *}, B\right)=0$. d) gives $\left(f_{a}(\lambda)=0 \wedge \lambda \in G_{k} \cap C^{-} \Rightarrow \lambda \in B\right)$, therefore $Z\left(\alpha, G_{k} \cap C^{-}\right)=0 . Z\left(\alpha, G_{k} \cap i R\right)=0$ because of $(15)$ and $\alpha>\alpha_{k}$. With a), we obtain $u>0$ and $2 k \pi+\pi<v<2 k \pi+2 \pi$ for the zero of $f_{\alpha}$ in $G_{k}, k \in N$, and $u>0$ and $\pi<|v|<2 \pi$ for the two zeros of $f_{\alpha}$ in $G_{0}$.

k) By Theorem 1, $f_{\alpha}$ has real zeros if and only if $\alpha \leq \alpha^{*}$, and the real zeros are negative. The assertion concerning the real zeros for $\alpha<\alpha^{*}$ follows from 
$Z\left(\alpha, G_{0}\right)=2$ and from the relations $u \in R \wedge f_{\alpha}(u)=0 \Leftrightarrow \alpha=g(u), u<u^{*} \Rightarrow g^{\prime}(u)>0$, $u>u^{*} \Rightarrow g^{\prime}(u)<0, g(0)=0, g(u) \rightarrow 0$ for $u \rightarrow-\infty, g \mid R^{-}>0$. $u^{*}$ must be a double zero of $f_{\alpha^{*}}$ since by $g\left(u^{*}\right)=\alpha^{*}=\max g$, there is no other real zero of $f_{\alpha^{*}}$, and the existence of a zero of $f_{\alpha^{*}}$ in $G_{0} \backslash R$ would imply the existence of a third zero in $G_{0}$, in contradiction to $Z\left(\alpha^{*}, G_{0}\right)=2$.

\section{Notation:}

$N, R, R^{+}, R^{-}$and $C$ denote the natural, real, positive real, negative real and the complex numbers respectively. We set $N_{0}:=N \cup\{0\}, R_{0}^{+}:=R \backslash R^{-}, R_{0}^{-}:=R \backslash R^{+}$. For $A \subset C, B \subset C$ and $z \in C$, we define

$$
A+z B:=\{\lambda \in C \mid \exists a \in A \exists b \in B: \lambda=a+z b\} .
$$

$\operatorname{Re} z(\operatorname{Im} z)$ is the real part (imaginary part) of $z$,

$$
\operatorname{Re} A:=\{u \in R \mid \exists z \in A: u=\operatorname{Re} z\}, \operatorname{Im} A:=\{u \in R \mid \exists z \in A: u=\operatorname{Im} z\} .
$$

$\partial A, \AA$ and $\bar{A}$ are the boundary, the interior and the closure of the set $A \subset C$. A dot - like in $\dot{n}$ - and a prime - like in $s^{\prime}$ - indicate differentiation. $C^{k}[-1,0]$ and $C^{k}(-1,0]$ are the sets of functions on $[-1,0]$ which have continuous derivatives up to order $k$ in $[-1,0]$ and $(-1,0]$ respectively. $f \mid A$ denotes the restriction of a given mapping $f: B \rightarrow D$ to a subset $A \subset B$.

\section{References}

[1] Dieudonne, J.: Foundations of modern analysis. New York: Academic Press 1960.

[2] Halbach, U., Burkhardt, H. J.: Sind einfache Zeitverzögerungen die Ursachen für periodische Populationsschwankungen? Oecologia (Berlin) 9, 215-222 (1972).

[3] Hutchinson, G. E.: Circular causal systems in ecology. Annals of the New York Academy of Sciences 50, 221-246 (1948).

[4] Hale, J. K.: Functional differential equations. Berlin-Heidelberg-New York: Springer 1971.

[5] Walther, H. O.: Asymptotic stability for some functional differential equations. Proceedings of the Royal Society of Edinburgh 74 A (1974/75).

[6] Wright, E. M.: A non-linear differential-difference equation. Jour. Reine Angewandte Math. $194,66-87(1955)$.

[7] Zygmund, A.: Trigonometric series I, Second edition. Cambridge: University Press 1959.

Dr. H.-O. Walther Mathematisches Institut Universität München Theresienstraße 39 D-8000 München 2 Federal Republic of Germany 